\title{
Transglutaminase 2 is dispensable but required for the survival of mice in dextran sulfate sodium-induced colitis
}

\author{
Eui Man Jeong ${ }^{1,2,5}$, Young Hoon Son ${ }^{1,5}$, Yewon Choi ${ }^{1}$, Jin-Hee Kim ${ }^{3,4}$, Jin-Haeng Lee ${ }^{1}$, Sung-Yup Cho ${ }^{1,2}$ and \\ In-Gyu Kim ${ }^{1,2}$
}

Transglutaminase 2 (TG2) is a ubiquitously expressed enzyme that catalyzes crosslinking, polyamination or deamidation of glutamine residues in proteins. It has been reported that TG2 is involved in the pathogenesis of various inflammatory diseases including celiac disease, pulmonary fibrosis, cystic fibrosis, multiple sclerosis and sepsis. Recently, using a mouse model of bleomycin-induced lung fibrosis, we showed that TG2 is required to trigger inflammation via the induction of T helper type 17 (Th17) cell differentiation in response to tissue damage. However, the role of TG2 in inflammatory bowel disease (IBD), which is thought to be a Th17 cell-associated disease, has remained elusive. In this study, we investigated the role of TG2 in dextran sulfate sodium (DSS)-induced colitis, the most widely used mouse model for IBD. Age- and sex-matched wild-type and TG2-Imice were fed $2 \%$ DSS for 7 days or 3.5\% DSS for 5 days in drinking water. An in situ TG activity assay revealed that DSS treatment activates TG2 in various colon cell types, including columnar absorptive cells and goblet cells. DSS-treated TG2-/mice showed lower interleukin (IL)-6, but higher IL-17A and ROR $\gamma \mathrm{t}$ (retinoic acid receptor-related orphan receptor- $\gamma \mathrm{t}$ ) expression levels in the colon tissues than that in the wild-type mice. Moreover, $\mathrm{TG}^{-/-}$mice showed higher mortality than the wild-type mice because of DSS treatment. Nevertheless, we found no significant differences in changes of body weight, colon length, morphology, immune cell infiltration and in vivo intestinal permeability between DSS-treated wild-type and TG2-/- mice. These results indicate that TG2-mediated Th17 cell differentiation is not required for the pathogenesis of DSS-induced acute colitis.

Experimental \& Molecular Medicine (2016) 48, e267; doi:10.1038/emm.2016.95; published online 4 November 2016

\section{INTRODUCTION}

Transglutaminase 2 (TG2) is a calcium-dependent enzyme that catalyzes transamidation reactions between glutamine residues of proteins and lysine residues of proteins, polyamines or $\mathrm{H}_{2} \mathrm{O}$, generating polyaminated, crosslinked or deamidated proteins. ${ }^{1}$ These TG2-mediated covalent modifications alter the activity of a number of proteins, such as RhoA, $\mathrm{I} \mathrm{I} \mathrm{B} \alpha$ and caspase 3 , resulting in modulation of cell adhesion, cytokine production and cell survival. ${ }^{2}$ TG2 is inactive under ordinary conditions, but is activated by oxidative stress provoked by tissue injury, inflammation or hypoxia. ${ }^{2-4}$ Thus, aberrant TG2 activation has been demonstrated to be implicated in the development of various oxidative stress-related diseases, including cataracts, fibrosis and cancer. ${ }^{1}$

Recently, a growing body of evidence has indicated that TG2 plays a critical role in triggering inflammation in several pathophysiological settings. ${ }^{1,5}$ In patients with celiac disease, TG2 generates deamidated gluten peptides in the small intestine that bind to human leukocyte antigen (HLA)-DQ2 and HLA-DQ8, HLA alleles associated with celiac disease, with a high affinity and then elicit strong T-cell activation that leads to intestinal inflammation. ${ }^{6}$ In addition, autoantibodies reactive to TG2 are detected in the serum of celiac patients. ${ }^{7}$ Analysis of autoantibodies revealed that new epitopes are exposed by self-crosslinking of TG2 with gluten peptide. The serum

\footnotetext{
${ }^{1}$ Department of Biochemistry and Molecular Biology, Seoul National University College of Medicine, Seoul, South Korea; ${ }^{2}$ Institute of Human-Environment Interface Biology, Seoul National University College of Medicine, Seoul, South Korea and ${ }^{3}$ Department of Anatomy, Seoul National University College of Medicine, Seoul, South Korea

${ }^{4}$ Current address: Department of Biomedical Laboratory Science, College of Health Science, Cheongju University, Cheongju, South Korea.

${ }^{5}$ These authors contributed equally to this work.

Correspondence: Professor I-G Kim, Department of Biochemistry and Molecular Biology, Seoul National University College of Medicine, 103 Daehak-ro, Jongno-gu, Seoul 110-799, South Korea.
}

E-mail: igkim@plaza.snu.ac.kr

Received 7 March 2016; revised 9 June 2016; accepted 13 June 2016 
autoantibody levels are well correlated with the severity of celiac disease, suggesting that aberrant TG2 activation elicits gluten-induced inflammation. Moreover, in patients with cystic fibrosis, defective CFTR (cystic fibrosis transmembrane regulator protein)-mediated oxidative stress upregulates TG2 that crosslinks and sequesters peroxisome proliferator-activated receptor- $\gamma$, a negative regulator for inflammatory gene expression, leading to increased production of proinflammatory cytokines and initiation of inflammation. ${ }^{8-10}$ Furthermore, in a mouse model of bleomycin-induced lung fibrosis, bleomycingenerated oxidative stress activates TG2 that in turn promotes interleukin (IL)-6 expression through activation of nuclear factor (NF)- $\mathrm{KB}$ in lung epithelial cells. A subsequent increase in IL-6 induces the differentiation of T helper type 17 (Th17) cells that amplify the inflammatory response via IL-17 secretion. ${ }^{11}$ Thus, TG2-mediated antigenic modification and/or Th17 cell differentiation seem to be crucial events in the pathogenesis of oxidative stress or tissue injury-induced inflammatory diseases.

Inflammatory bowel disease (IBD), comprising Crohn's disease and ulcerative colitis, is an immune-mediated inflammatory disorder mainly affecting the small and large intestine. ${ }^{12,13}$ It has been widely appreciated that polygenic predisposition combined with complex interactions among immune cells, intestinal epithelial cells and luminal substances, such as enteric bacteria and dietary antigens, significantly contribute to the etiology of gastrointestinal inflammation. ${ }^{12,13}$ Thus far, genome-wide association studies have identified more than 160 loci containing IBD susceptibility genes. ${ }^{14}$ Intriguingly, pathway analyses of these genes revealed a highly significant association between IBD and the IL-23 signaling pathway (IL23R, TYK2, JAK2, STAT3 and CCR6) as well as the NF- $\mathrm{KB}$ signaling pathway (REL, TNFAIP3 and NFKB1), emphasizing that Th17 cell differentiation is importantly implicated in triggering the intestinal inflammation of IBD. ${ }^{15-17}$ Moreover, significantly high levels of TG2 autoantibody have been detected in the serum of Crohn's disease and ulcerative colitis patients, ${ }^{18}$ indicating that TG2 might be activated in the inflamed intestine of IBD. Therefore, based on these observations, we hypothesized that aberrant TG2 activation might be involved in the pathogenesis of IBD by inducing Th17 cell differentiation.

Oral consumption of dextran sulfate sodium (DSS) in drinking water induces colitis in many experimental animals, including mouse, rat, guinea pig and hamster, resembling the clinical and histological features of IBD, particularly ulcerative colitis. ${ }^{19}$ DSS is thought to induce colitis by damaging intestinal epithelial cells, leading to penetration of enteric commensal bacteria into epithelial cells that triggers intestinal inflammation, ${ }^{20}$ and/or by directly activating dendritic cells. ${ }^{21}$ The intensity of colitis depends on the concentration and duration of DSS administration, and can be mitigated by withdrawal of DSS from drinking water to mimic remission of IBD. ${ }^{19}$ In this study, we thus investigated the role of TG2 in IBD pathogenesis using a mouse model of DSS-induced colitis. Our results show that administration of DSS activates TG2 in the mouse colon. However, contrary to our hypothesis, there was no significant difference in DSS-induced colitis between wild-type and $\mathrm{TG}^{-1-}$ mice. Notably, with extended periods of DSS administration, $\mathrm{TG}^{-1-}$ mice exhibit higher mortality rates than wild-type mice. Our results indicate that TG2 has no crucial role in acute DSS-induced colitis.

\section{MATERIALS AND METHODS}

\section{Mice}

C57BL/6J mice were purchased from The Jackson Laboratory (Bar Harbor, ME, USA). TG2 $2^{-1-}$ mice were received from Dr Gerry Melino (University Tor Vergata, Rome, Italy) ${ }^{22}$ and backcrossed with C57BL/6J mice for 12 generations. Mice were housed in a specific pathogen-free environment at the animal facility of Seoul National University College of Medicine (Seoul, South Korea). All animal experiments were conducted with the approval of the institutional animal care and use committee at Seoul National University (SNU-101220-6).

\section{Induction of colitis by DSS}

Wild-type and $\mathrm{TG}^{-1-}$ mice were kept under a thermostatic and photoperiodic control $\left(25^{\circ} \mathrm{C}\right.$ and $12: 12 \mathrm{~h}$ light/dark cycle, respectively). They were provided access to gluten-free standard mouse chow and filtered tap water ad libitum for at least 14 days to adapt to these conditions before inclusion in the experiments. DSS (36 000-50 000 Da; MP Biomedicals, Santa Ana, CA, USA) was dissolved in autoclaved water (2 and $3.5 \% \mathrm{w} / \mathrm{v})$. Colitis was induced by feeding 2 or $3.5 \%$ DSS for the indicated number of days to age- and sex-matched mice (aged 14 weeks) as described previously, and mean water consumption was monitored for each group. ${ }^{23}$

For the survival study, the mice were checked each day for signs of morbidity, and their body weights were recorded. Mice were determined to be dead if they did not respond to a gentle touch with a platinum wire pick; they were immediately killed with $\mathrm{CO}_{2}$. The end points of the survival experiments were determined by the changes in body weight. Mice treated with 2\% DSS for 7 days were killed by $\mathrm{CO}_{2}$ asphyxiation on day 21 when the body weight of mice from all the groups appeared to have recovered to the initial baseline value. Mice treated with $2 \%$ DSS for 8 days or 3.5\% DSS for 6 days were killed on day 10 when mice from all the groups exhibited the maximum loss of body weight.

\section{Immunohistochemistry on cryosections}

Mice were intraperitoneally injected with biotin-amido-pentylamine $\left(100 \mathrm{mg} \mathrm{kg}^{-1}\right)$. After $3 \mathrm{~h}$, mice were killed by $\mathrm{CO}_{2}$ asphyxiation. The colons were harvested, embedded in Tissue-Tek OCT compound (Sakura Finetek Inc., Torrance, CA, USA) and snap-frozen in liquid nitrogen. Frozen sections $(10 \mu \mathrm{m})$ were cut from OCT-embedded tissues and fixed in cold acetone for $10 \mathrm{~min}$ and dried in air for $1 \mathrm{~h}$. The sections were blocked with $\mathrm{H}_{2} \mathrm{O}_{2}$ and subsequently treated with $10 \%$ goat serum at room temperature for $20 \mathrm{~min}$. They were then incubated with anti-TG2 polyclonal antibody (Ab4, Thermo Fisher Scientific, Fremont, CA, USA) at $4{ }^{\circ} \mathrm{C}$ for $18 \mathrm{~h}$, followed by staining TG2 protein with Alexa Fluor 488-conjugated goat anti-rabbit IgG $(\mathrm{H}+\mathrm{L})$ secondary antibody (Molecular Probes Inc., Eugene, OR, USA) in accordance with the manufacturer's instructions. Biotin-amidopentylaminylated proteins were detected with Texas Red-conjugated streptavidin (Molecular Probes) to measure TG activity. The sections were mounted with fluorescent mounting medium (Dako North America Inc., Carpinteria, CA, USA) and observed using a FluoView 1000 confocal microscope (Olympus, Tokyo, Japan). 


\section{Gross and histopathological evaluation of colon}

Mice were monitored for body weight, stool consistency, rectal bleeding, hunched posture and mortality every day or every other day during DSS treatment. After 5 or 10 days of DSS-water feeding, mice were killed with $\mathrm{CO}_{2}$. The entire colon from cecum to anus was dissected, and its length was measured. The colon was incised and flushed with cold phosphate-buffered saline to remove fecal material. Proximal and distal colons were fixed with $4 \%$ paraformaldehyde, embedded in paraffin and stained with hematoxylin and eosin for morphological evaluation as described previously. ${ }^{23}$

\section{Myeloperoxidase (MPO) assay}

MPO activity was assessed to evaluate the extent of neutrophil infiltration into the inflamed colon. Colon specimens were homogenized with 20 volumes of $50 \mathrm{~mm}$ phosphate buffer ( $\mathrm{pH}$ 6.0) containing $0.5 \%$ hexadecyltrimethyl ammonium bromide (SigmaAldrich, St Louis, MO, USA) using Precellys 24 (Bertin Technologies, Montigny-le-Bretonneux, France) and sonicated for $10 \mathrm{~s}$. Homogenates were freeze-thawed three times, and then centrifuged for $15 \mathrm{~min}$ $(14000 \mathrm{~g})$ at $4{ }^{\circ} \mathrm{C}$. Supernatant $(14 \mu \mathrm{l})$ was mixed with $1 \mu$ of substrate solution ( $1 \mathrm{mg} \mathrm{ml}^{-1}$ o-dianisidine hydrochloride and $0.0005 \%$ hydrogen peroxide; Sigma-Aldrich). The absorbance at $460 \mathrm{~nm}$ was monitored using a microplate spectrophotometer (Molecular Devices, Sunnyvale, CA, USA). One unit of MPO activity was defined as the absorbance change per min at $25^{\circ} \mathrm{C}$ in the reaction with $1 \mu \mathrm{mol}$ horseradish peroxidase.

\section{In vivo intestinal permeability assay}

In vivo permeability assays were performed using fluorescein isothiocyanate (FITC)-dextran as a permeability tracer, as described previously with slight modification. ${ }^{23}$ Briefly, mice were housed without food and water for $4 \mathrm{~h}$ and then gavaged with FITC-dextran (0.6 $\mathrm{g} \mathrm{kg}^{-1}$, molecular weight 3000-5000 Da; Sigma-Aldrich). After $4 \mathrm{~h}$, mice were killed and blood samples were taken. Fluorescence intensity of serum was measured by using an Infinite M200 (excitation at $492 \mathrm{~nm}$ and emission at $525 \mathrm{~nm}$; Tecan, Männedorf, Switzerland). FITC-dextran concentrations in serum were calculated from standard curves generated by serial dilution of FITC-dextran $(0,64,320,1600$, 8000 and $40000 \mathrm{ng} \mathrm{ml}^{-1}$ ).

\section{Real-time PCR}

Total RNA was extracted from the distal colon using TRIzol (Invitrogen, Carlsbad, CA, USA) and then reverse transcribed using SuperScript II Reverse Transcriptase (Invitrogen). Real-time PCR was performed with a KAPA SYBR FAST qPCR Kit (Kapabiosystems, Cape Town, South Africa) using a CFX96TM Real-Time system (Bio-Rad, Hercules, CA, USA). The following specific primers were used: 34B4, 5'-TCCAGGCTTTGGGCATCA-3' (forward) and 5'-CTTTATCAGC TGCACATCACTCAGA-3' (reverse); tumor necrosis factor- $\alpha$ (TNF $\alpha$ ), 5'-AGGCTGCCCCGACTACGT-3' (forward) and 5'-GACTTTCTCC TGGTATGAGATAGCAAA- $3^{\prime}$ (reverse); interferon- $\gamma$ (IFN $\gamma$ ), $5^{\prime}$-CAG CAACAGCAAGGCGAAA- $3^{\prime}$ (forward) and $5^{\prime}$-CTGGACCTGTGGGT TGTTGAC-3' (reverse); IL-1 $\beta, 5^{\prime}$-TCGCTCAGGGTCACAAGAAA-3' (forward) and 5'-CATCAGAGGCAAGGAGGAAAAC-3' (reverse); IL-6-ACAAGTCGGAGGCTTAATTACACAT-3' (forward) and 5'-TGC CATTGCACAACTCTTTT-3' (reverse); KC, 5'-CTTGAAGGTGTTG CCCTCAG- $3^{\prime}$ (forward) and $5^{\prime}$-TGGGGACACCTTTTAGCATC- ${ }^{\prime}$ (reverse); IL-10, 5'-GGTTGCCAAGCCTTATCGGA-3' (forward) and 5'-ACCTGCTCCACTGCCTTGCT-3' (reverse); IL-12, 5'-AGACCCT GCCCATTGAACTG-3' (forward) and $5^{\prime}$-GAAGCTGGTGCTGTAG
TTCTCATATTT-3' (reverse); 5'-GGCAAGGCTAACTGACCTGGA AAGG-3' (forward) and 5'-ACAGCGAGGCACATCAGGTACGA-3' (reverse). ${ }^{23}$ mRNA expression levels were determined by the $2^{-\Delta \Delta \mathrm{Ct}}$ method $^{24}$ and normalized or compared with $34 \mathrm{~B} 4$ levels. The results are presented as relative fold change compared with control samples.

\section{Statistical analysis}

$P$-values were calculated with Prism 5 software (GraphPad, San Diego, CA, USA) using two-way analysis of variance followed by Bonferroni post tests to compare individual means for body weight changes, colon length, MPO activity, FITC-dextran permeability and real-time PCR. $P$-values of the survival rate data were analyzed by using the log-rank test.

\section{RESULTS}

DSS induces and activates TG2 in mouse colon

We first examined the effects of DSS administration on TG2 expression and activity in the colon. Wild-type and TG2 $2^{-1-}$ mice were administered DSS for 5 days, and then treated intraperitoneally with biotinylated pentylamine. After killing of mice, cryosections of the colon were stained with an anti-TG2 antibody or Texas Red-conjugated streptavidin for determining TG2 protein levels or in situ TG activity, respectively. In wildtype mice not exposed to DSS, TG2 was majorly expressed in the lamina propria, muscularis mucosae and submucosa of the colon, and weakly expressed in columnar absorptive cells and goblet cells (Figure 1, no treatment, TG2 ${ }^{+/+}$). However, no TG activity was detected in the colon of these mice. In contrast, treatment with $3.5 \%$ DSS for 5 days caused a substantial increase in the levels of TG2 protein compared with mice not exposed to DSS. Moreover, high TG activity was observed in the colon of DSS-treated mice. Intriguingly, columnar absorptive cells showed relatively high TG activity in spite of low levels of TG2 expression (Figure 1, 3.5\% DSS, $\mathrm{TG}^{+/+}$and Supplementary Figure 1). As expected, in $\mathrm{TG} 2^{-1-}$ mice treated with DSS, no TG2 protein or activity was detected, demonstrating that TG2 is the major TG isoenzyme expressed in the colon, and contributes to DSS-induced TG activity (Figure 1, 3.5\% DSS, TG2 $2^{-1-}$ ). These results indicate that DSS increases TG2 expression and activates TG2 in mouse colon.

\section{TG2 is not required for the development of DSS-induced colitis in mouse}

To test whether TG2 is involved in DSS-induced acute colitis, we compared the severity of colitis in wild-type mice with that of $\mathrm{TG}^{-/-}$mice by monitoring body weight after administration of $2 \%$ DSS in drinking water for 7 days. We observed that there were no significant differences in water intake, the extent of diarrhea and rectal bleeding between wild-type and TG2 $2^{-1-}$ mice (data not shown). Body weight of both mouse groups decreased similarly from day 7 of DSS administration, to the lowest level at day 9, and then began to recover to the level of untreated mice (Figure 2a). Under these experimental conditions, there was no significant difference in survival rate between wild-type and $\mathrm{TG}^{-/-}$mice (Figure $2 \mathrm{~b}$ ). We then evaluated the effect of extending the period of DSS administration to 8 days or increasing the DSS concentration to $3.5 \%$ 


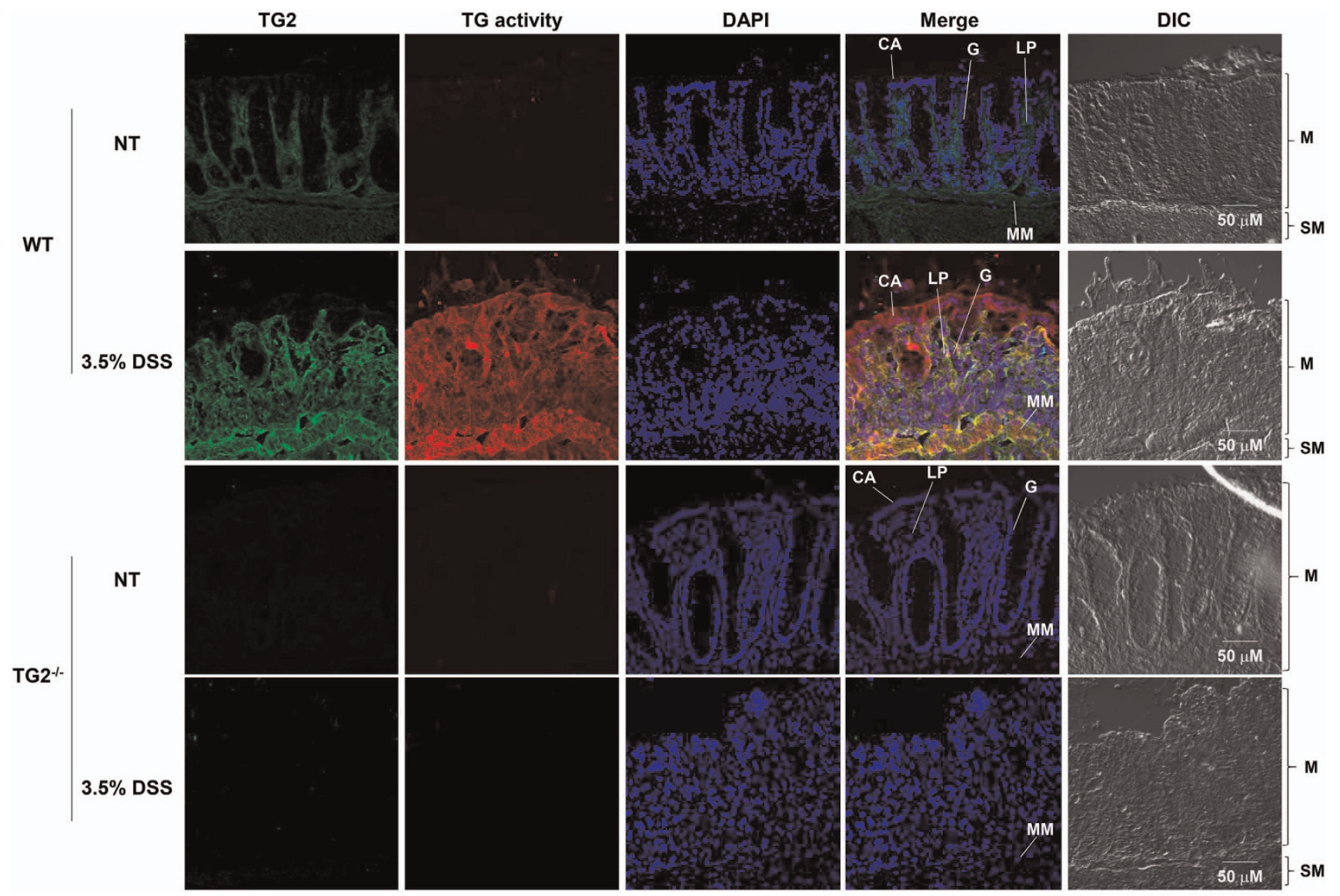

Figure 1 Transglutaminase 2 (TG2) expression and in vivo activity in colon from dextran sulfate sodium (DSS)-treated mice. Wild-type (WT) and $\mathrm{TG}^{-/-}$mice were treated with $3.5 \%$ DSS in drinking water for 5 days, and were intraperitoneally injected with biotin-amidopentylamine $\left(100 \mathrm{mg} \mathrm{kg}^{-1}\right)$. After $3 \mathrm{~h}$, mice were killed. Cryosections of colon were stained with anti-TG2 antibody, Texas Red-conjugated streptavidin and DAPI for TG2 protein, TG activity and nucleus detection, respectively. Data are representative of three experiments. CA, columnar absorptive cells; DAPI, 4',6-diamidino-2-phenylindole; DIC, differential interference contrast; G, goblet cells; LP, lamina propria; M, mucosa; MM, muscularis mucosae; NT, no treatment; SM, submucosa.

on the severity of colitis. In both experimental settings, however, there was no significant difference in body weight changes between wild-type and $\mathrm{TG}^{-1-}$ mice (Figure 2c). In contrast, $\mathrm{TG}^{-/-}$mice showed significantly higher mortality rates under these conditions compared with wild-type mice (Figure 2d).

To confirm these results, we examined the colons of mice administered 2\% DSS for 8 days or 3.5\% DSS for 5 days. Colon length of both wild-type and $\mathrm{TG} 2^{-1-}$ mice was significantly reduced to $\sim 65 \%$ and $85 \%$ of control mice by exposure to $2 \%$ and $3.5 \%$ DSS, respectively, but there was no difference in colon length between wild-type and TG2 ${ }^{-1-}$ mice (Figures $3 \mathrm{a}-\mathrm{c}$ ). Histological analysis revealed that proximal and distal colons exposed to DSS showed a loss of crypts and extensive infiltration of immune cells throughout the mucosa. The inflammatory response was more pronounced in distal colon than proximal colon, but there was no significant difference in pathological features between wild-type and $\mathrm{TG}^{-1-}$ mice (Figures $4 \mathrm{a}$ and $\mathrm{b}$ ). These results indicate that TG2 is not involved in the pathogenesis of DSS-induced colitis, but is required for survival in inflammatory environments.

\section{TG2 activation is not associated with epithelial barrier function}

TG2 is known to be involved in extracellular matrix formation by virtue of its protein crosslinking activity that contributes to epithelial barrier function. ${ }^{25}$ In DSS-induced colitis, it has been suggested that the intestinal epithelial barrier is disrupted, thereby increasing penetration of enteric bacteria and inflammation. ${ }^{20}$ Thus, we evaluated the epithelial barrier function of DSS-exposed wild-type and $\mathrm{TG}^{-1-}$ mice by assaying the in vivo transepithelial permeability of the colon, as described previously. ${ }^{23}$ FITC-dextran was administered to DSS-treated mice by intragastric gavage, and its concentration in serum was determined by fluorescence spectrometry. Levels of FITC-dextran in the serum of 2 or 3.5\% DSS-treated mice were approximately five fold higher than those of control mice, but there was no significant difference in FITC-dextran levels between wild-type and $\mathrm{TG}^{-/-}$mice (Figure $5 \mathrm{a}$ ). To further confirm these results, we quantified the extent of neutrophil infiltration into the mucosa by determining MPO activity in the colon. Administration of DSS significantly increased the levels of MPO activity in the distal colon of both wild-type and 
a

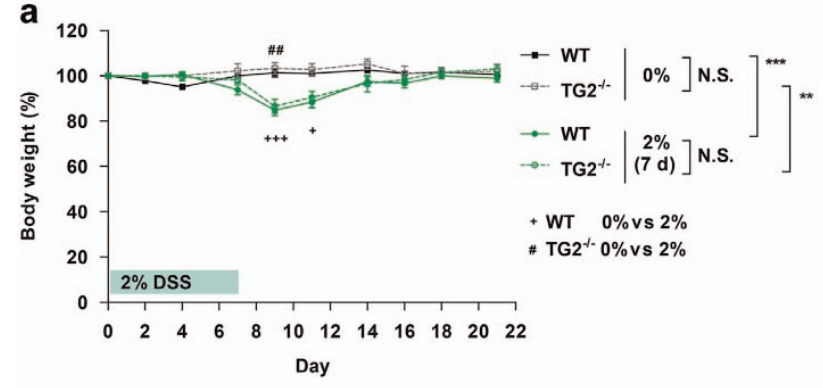

C

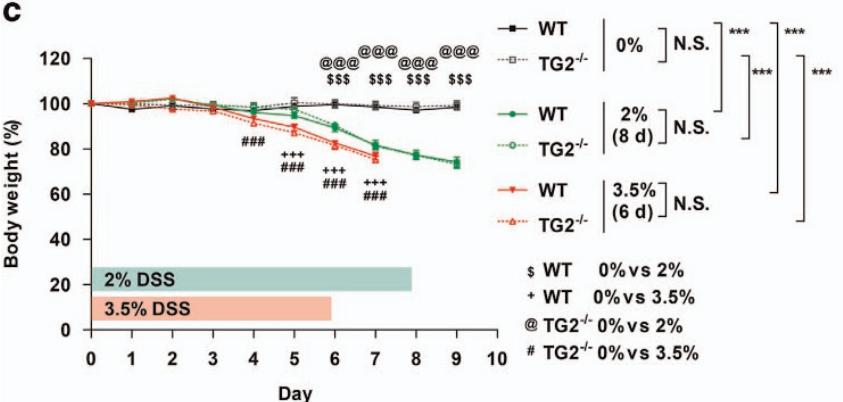

b

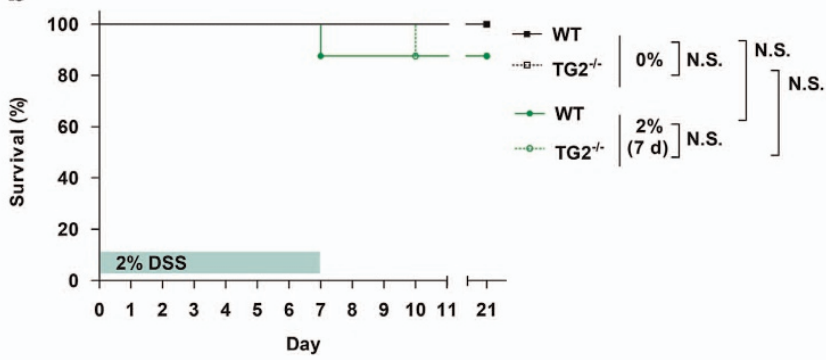

d

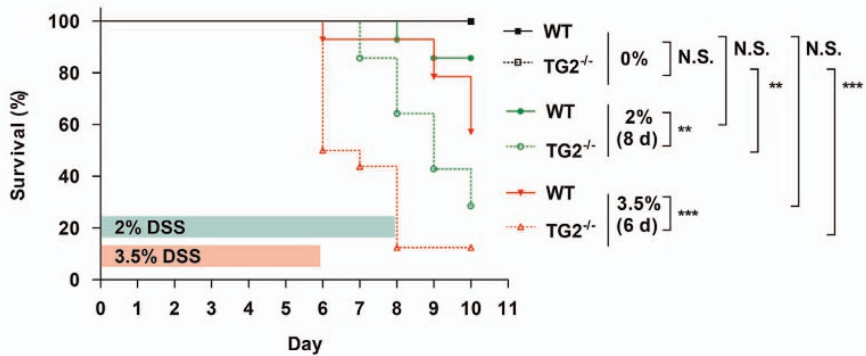

Figure 2 Effects of dextran sulfate sodium (DSS) treatment on body weight and survival of wild-type (WT) and transglutaminase 2-deficient $\left(\mathrm{TG} 2^{-l-}\right.$ ) mice. (a, b) WT and TG2 ${ }^{-1-}$ mice (14-week-old females) were fed ad libitum water containing $0 \%(n=2)$ and $2 \%(n=8)$ DSS for 7 days. (c, d) Age- and sex-matched WT and TG2 ${ }^{-1-}$ mice were fed ad libitum water containing $2 \%(n=14)$ and $3.5 \%(n=15)$ DSS for 8 and 6 days, respectively, or plain water $(n=6)$. (a, c) Body weight was analyzed in the mice survived at the indicated time points and the value expressed as percentage of weight on the day of first exposure to DSS is plotted against time. Data are presented as the mean \pm s.e.m. Overall $P$-values analyzed using two-way analysis of variance (ANOVA) are shown at the right; individual Bonferroni post-test results are shown above the line profiles. (b, d) Survival curves of WT and TG2 ${ }^{-1-}$ mice are depicted against time. The $P$-values were calculated using the log-rank test. ${ }^{*} P<0.05 ;{ }^{*} P<0.01 ;{ }^{* *} P<0.001$; NS, not significant.

$\mathrm{TG}^{-1-}$ mice, but no difference in MPO activity was observed between wild-type and TG2 ${ }^{-1-}$ mice (Figure $5 \mathrm{~b}$ ). These results indicate that TG2 is not involved in the modulation of barrier function in response to DSS treatment.

\section{TG2 modulates colon tissue-specific expression of inflammatory mediators}

TG2 plays a crucial role in eliciting an inflammatory response to tissue damage in a mouse model of bleomycininduced lung fibrosis. ${ }^{11}$ TG2-mediated IL- 6 production in damaged lung epithelial cells induces Th17 cell differentiation, triggering inflammation through IL-17. In contrast, as shown in the results described above, TG2 is not required for an inflammatory response in the DSS-induced colitis model.

To obtain a possible explanation for the different role of TG2 in intestinal inflammation, we monitored the mRNA levels of inflammatory cytokines in the distal colon of mice administered 3.5\% DSS using real-time PCR. DSS exposure markedly increased the expression of IL-6 at day 5, and its levels in $\mathrm{TG} 2^{-1-}$ mice were significantly lower than those in wild-type mice (Figure 6a). In the same colon tissues, levels of IL-17A were increased at day 3 after DSS treatment in both mice, but significantly higher in $\mathrm{TG} 2^{-1-}$ mice than wild-type mice (Figure 6b), whereas levels of IL-17F were similarly changed by DSS treatment in both mice (Figure 6c). Moreover, levels of ROR $\gamma \mathrm{t}$, a master regulatory transcription factor in
Th17 cells, were increased more obviously in $\mathrm{TG}^{-/-}$mice than wild-type mice at day 3 (Figure $6 \mathrm{~d}$ ). These results suggest that TG2 suppresses, rather than induces as in lung tissue, the differentiation of IL-17A-secreting Th17 cells or group 3 innate lymphoid cells in the mouse colon.

$\mathrm{TNF} \alpha$ and keratinocyte chemoattractant $(\mathrm{KC})$ are known to be inflammatory cytokine/chemokines associated with IBD. ${ }^{26,27}$ Levels of both mediators were gradually increased during DSS treatment, and their levels in TG2 $2^{-1-}$ mice were significantly higher than those in wild-type mice (Figures $6 \mathrm{e}$ and $\mathrm{f}$ ). Paradoxically, expression levels of IL-10 in $\mathrm{TG}^{-/-}$mice, a well-known anti-inflammatory cytokine in IBD, were significantly higher than those in wild-type mice at day 3 (Figure 6g). In addition, DSS treatment increased the expression of IL-12 and IFN $\gamma$ in both wild-type and TG2 $2^{-/-}$ mice, but had no effect on IL-1 $\beta$ mRNA level (Figures $6 \mathrm{~h}-\mathrm{j}$ ). Taken together, these results indicate that TG2 modulates the expression of inflammatory mediators in an intestine-specific manner in response to tissue damage.

\section{DISCUSSION}

TG2 has been reported to participate in the pathogenesis of various inflammatory diseases including lung fibrosis, cystic fibrosis, multiple sclerosis and sepsis. ${ }^{1}$ However, the role of TG2 in IBD has remained elusive. In the present study, using TG2-deficient mice, we examined the role of TG2 in the 

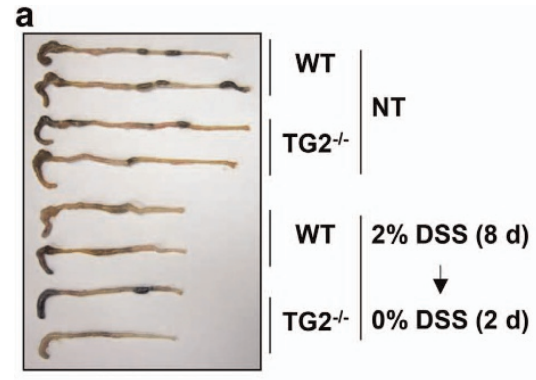

b

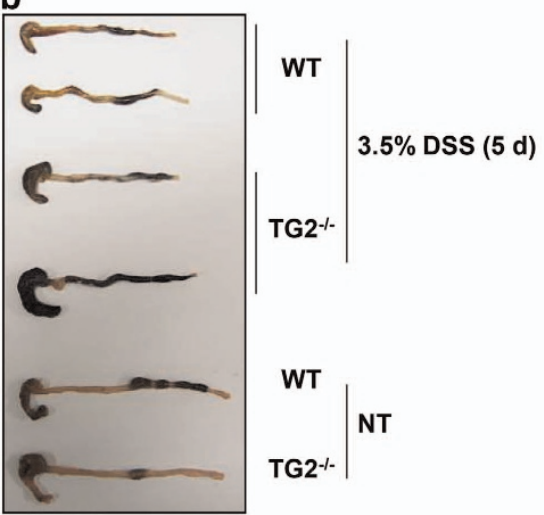

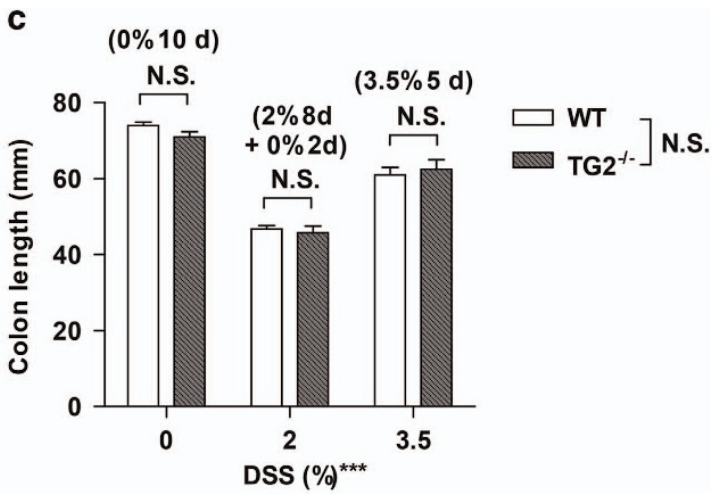

Figure 3 Gross changes of colon from dextran sulfate sodium (DSS)-treated wild-type (WT) and transglutaminase 2-deficient (TG2 ${ }^{-/-}$) mice. (a, b) Age- and sex-matched wild-type and TG2 ${ }^{-1-}$ mice were treated with $2 \%$ DSS water for 8 days and water for an additional 2 days (a) or 3.5\% DSS water for 5 days (b). After mice were killed, colons were separated and photographed ( $n=4$ for each group). (c) Length of colon was measured in the survived mice and graphed as the mean \pm s.e.m. values. Overall $P$-values analyzed using two-way analysis of variance (ANOVA) are shown; individual Bonferroni post-test results are shown above the columns. ${ }^{* * *} P<0.001$; NS, not significant; NT, no treatment.
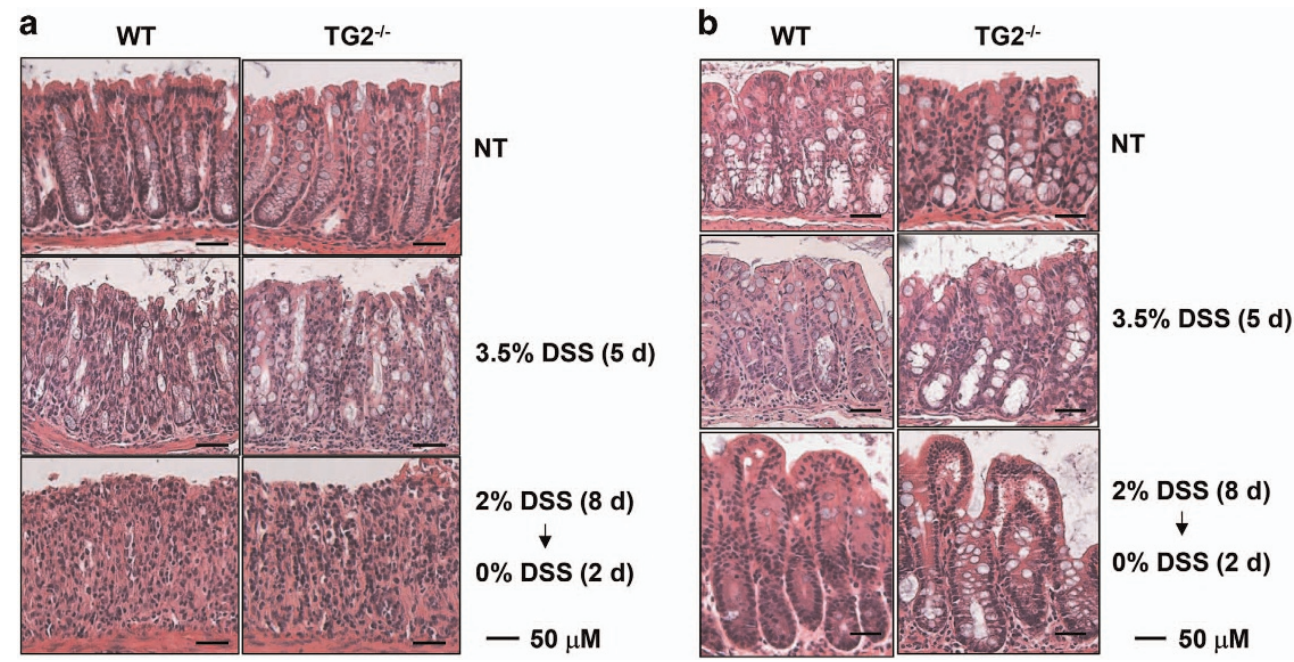

Figure 4 Histopathological changes of colon from dextran sulfate sodium (DSS)-treated wild-type (WT) and transglutaminase 2-deficient $\left(\mathrm{TG} 2^{-/-}\right.$) mice. Age- and sex-matched WT and TG2 ${ }^{-/-}$mice were treated with $2 \%$ DSS water for 8 days and water for an additional 2 days or 3.5\% DSS water for 5 days. Representative hematoxylin and eosin-stained sections of distal colon (a) and proximal colon (b) from DSS-treated mice. Data are representative of three experiments; NT, no treatment.

process of DSS-induced acute colitis. Our results show that there were no significant differences between DSS-exposed wild-type and TG2 ${ }^{-1-}$ mice in loss of body weight, shortening of colon, histological changes, immune cell infiltration or in vivo intestinal permeability. These results indicate that
TG2 is not required for inducing acute colitis in DSS-administered mice.

It is widely appreciated that intracellular TG2 is inactive under normal conditions, but is activated by injury to the extracellular matrix or plasma membrane. ${ }^{3}$ In the DSS-induced 


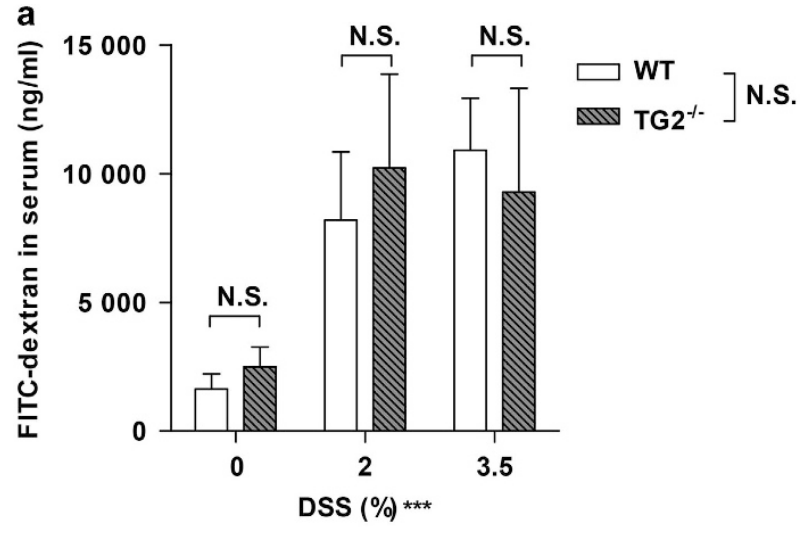

b

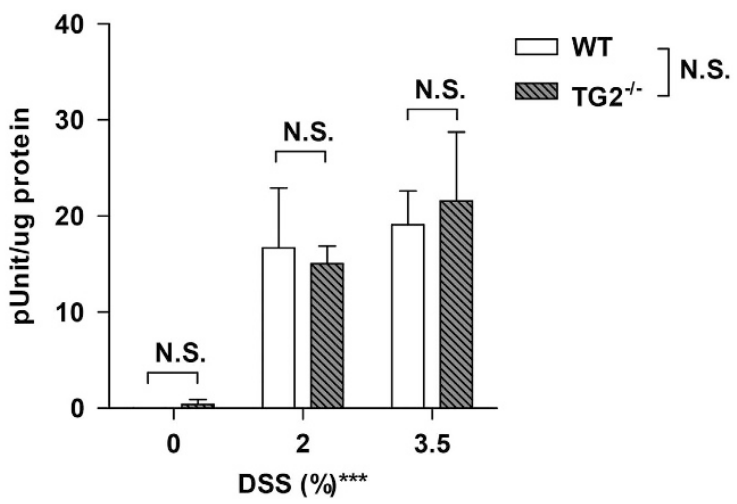

Figure 5 In vivo intestinal permeability and myeloperoxidase activity in colon of dextran sulfate sodium (DSS)-treated wild-type (WT) and tansglutaminase 2-deficient (TG2 ${ }^{-/-}$) mice. Age- and sex-matched WT and TG2 ${ }^{-1-}$ mice were administered $2 \%$ DSS water for 7 days and water for an additional 3 days or 3.5\% DSS water for 5 days. (a) Mice were gavaged with fluorescein isothiocyanate (FITC)-dextran (60 mg per $100 \mathrm{~g}$ of body weight), and serum FITC-dextran levels were quantified and graphed as the mean and s.d. values for each group ( $n=3$ for each group). (b) Mice were killed, and myeloperoxidase activity was measured in distal colons. The activity was graphed as the mean \pm s.e.m. values for each group ( $n=3$ for each group). Data are representative of at least two experiments. Overall $P$-values analyzed using two-way analysis of variance (ANOVA) are shown; individual Bonferroni post-test results are shown above the columns. ${ }^{* *} P<0.001$; NS, not significant.

colitis model, DSS is known to be directly toxic to intestinal epithelial cells. ${ }^{20}$ Thus, TG2 is considered to be activated by DSS-induced colon injury in mouse. However, our data demonstrate that there is no causal relationship between TG2 activity and DSS-induced intestinal inflammation, suggesting that TG2 activation might be a consequence, not a cause, of DSS-induced inflammation. Indeed, it has been reported that intracellular TG2 is also activated by treatment with polyinosinic:polycytidylic acid (poly(I:C)), an agonist for Toll-like receptor $3,{ }^{28}$ and that DSS treatment results in disruption of the mucosal barrier and increased mucosal permeability, thereby allowing enteric commensal bacteria to penetrate into the mucosa. ${ }^{20}$ Therefore, bacterial components might initiate the inflammatory responses, but also concomitantly induce the activation of intracellular TG2, explaining TG2 activity-independent colon inflammation.

Interestingly, similar findings have been reported in a mouse model of poly(I:C)-induced enteropathy. Poly(I:C) treatment activates TG2 in villus tips of the small intestine, but TG2 activity is not correlated with severity of villous atrophy. However, in gliadin-sensitive NOD-DQ8 mice, TG2 is proposed to be responsible for dietary gliadin-induced exacerbation of poly(I:C)-triggered tissue damage in the small intestine, ${ }^{28}$ suggesting that TG2 activity may be involved in the modulation of cellular responses to inflammatory cues. Thus, it is plausible that DSS-mediated enteropathy might be aggravated or alleviated by intestinal TG2 activity only under specific conditions.

Our results show that $\mathrm{TG}^{-1-}$ mice are more susceptible to DSS treatment than wild-type mice. Previously, mixed results on the susceptibility of $\mathrm{TG} 2^{-/-}$mice to treatment with various agents have been reported. $\mathrm{TG}^{-1-}$ mice are more resistant to lipopolysaccharide than wild-type mice, ${ }^{10,29}$ whereas $\mathrm{TG}^{-/-}$ mice are more susceptible to TNF $\alpha$ /actinomycin D, carbon tetrachloride and poly(I:C) than wild-type mice. ${ }^{28-30}$ Although the mechanism underlying these differences in susceptibility is presently unclear, the type of target tissue and extent of systemic effects have been proposed to significantly contribute to the susceptibility. For example, lipopolysaccharide-triggered, TG2-mediated cytokine production in macrophages is thought to increase the mortality of wild-type mice, but not of $\mathrm{TG}^{-1-}$ mice, by inducing systemic organ damage. ${ }^{10,29}$ In the cases of carbon tetrachloride and TNF $\alpha /$ actinomycin $\mathrm{D}$, it has been suggested that TG2 protects the liver from toxic effects rather than eliciting systemic inflammatory responses. ${ }^{29,30}$ In contrast, in DSS-induced colitis and poly(I:C)-induced enteropathy, there is no significant difference in intestinal inflammation. ${ }^{28}$ Thus, the susceptibility of $\mathrm{TG}^{-1-}$ mice may depend on systemic toxic effects of these drugs.

DSS penetrates into the epithelial cell layer in the intestine in its intact form, ${ }^{31}$ and is primarily eliminated by Kupffer cells in the liver and by auxiliary macrophages in the spleen through phagocytosis. $^{32}$ Because TG2-deficient macrophages showed a reduced capacity for phagocytic uptake of apoptotic cells, ${ }^{33}$ as well as low levels of the gp91 ${ }^{\text {phox }}$ subunit of the phagocytic $\mathrm{NADPH}$ oxidase (nicotinamide adenine dinucleotide phosphate-oxidase) that destroys invading bacteria, cell debris and foreign materials in neutrophils, ${ }^{34}$ it is possible that DSS could not be efficiently removed but accumulated in the liver or spleen in TG2-deficient mice, resulting in DSS-induced liver or spleen tissue damage and increased mortality. Similarly, in the case of poly(I:C)-induced enteropathy, intraperitoneally administered poly(I:C) can be distributed systemically and induce tissue damage in the liver and brain, leading to increased mortality of TG2 ${ }^{-1-}$ mice. ${ }^{28,35,36}$ However, we could find no difference in the post-mortem histology of the liver, kidney, spleen, heart and colon of wild-type mice and TG2-lmice following DSS treatment (Supplementary Figure 2). Thus, further studies are required for elucidating the cause of increased DSS-induced mortality in $\mathrm{TG}^{-1-}$ mice. 

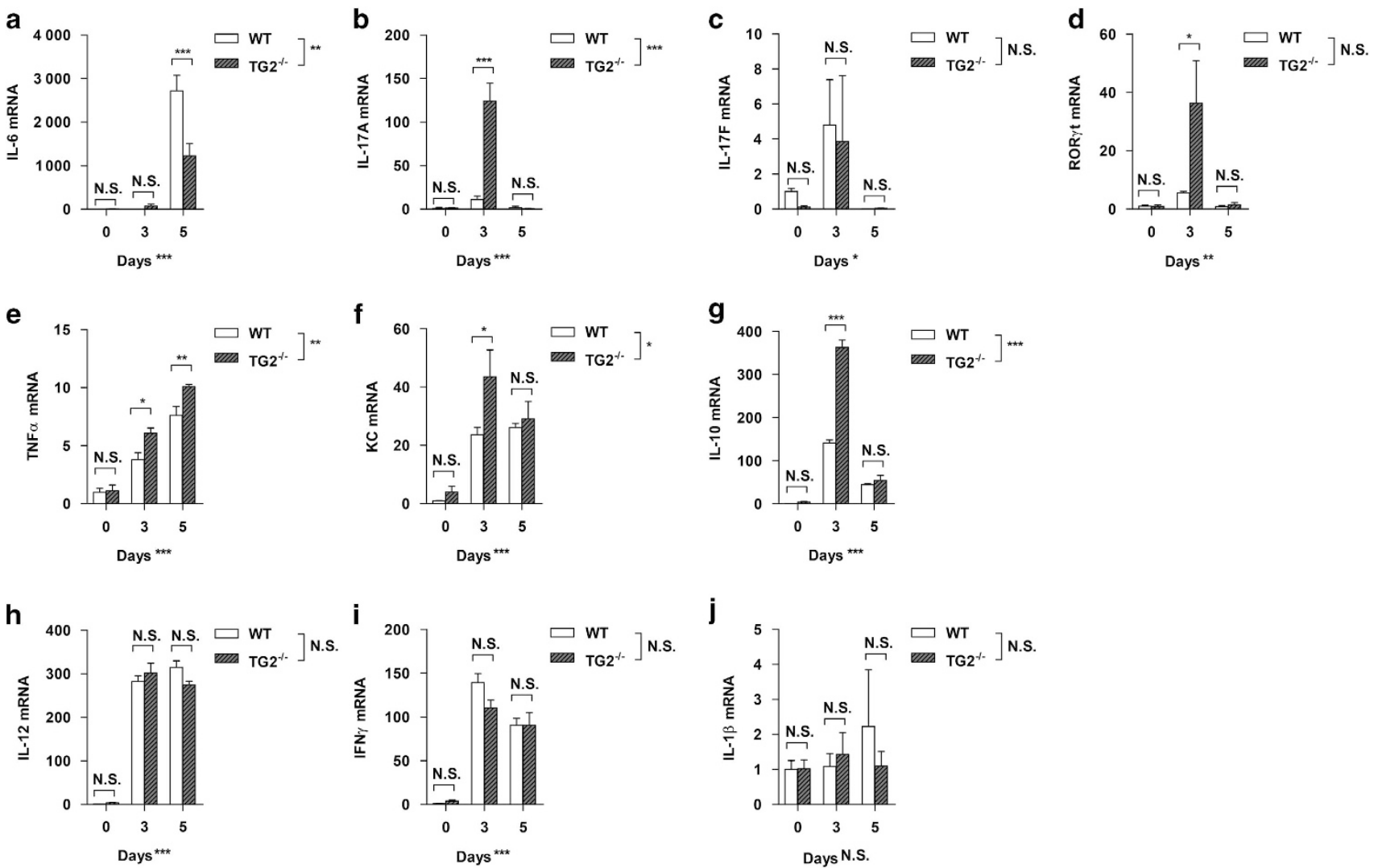

Figure 6 Comparison of expression levels of inflammatory mediators in colon between dextran sulfate sodium (DSS)-treated wild-type (WT) and transglutaminase 2-deficient (TG2 ${ }^{-1-}$ ) mice. WT and TG2 ${ }^{-l-}$ mice were treated with 3.5\% DSS water for 3 or 5 days. Total RNA was prepared from distal colon and reverse transcribed. Using real-time PCR, relative mRNA levels of (a) interleukin (IL)-6, (b) IL-17A, (c) IL-17F, (d) retinoic acid receptor-related orphan receptor- $\gamma \mathrm{t}$ (ROR $\gamma \mathrm{t}$ ), (e) tumor necrosis factor- $\alpha$ (TNF $\alpha$ ), (f) keratinocyte chemoattractant (KC), (g) IL-10, (h) IL-12, (i) interferon- $\gamma$ (IFN $\gamma$ ) and (j) IL-1 $\beta$ were determined and graphed as the mean \pm s.e.m. values for each group. Data are representative of at least two experiments. Overall $P$-values analyzed using two-way analysis of variance (ANOVA) are shown; individual Bonferroni post-test results are shown above the columns ( $n=3$ for each group). ${ }^{*} P<0.05$; ${ }^{* *} P<0.01$; $* * * P<0.001$; NS, not significant.

The regulation of cytokine expression makes a critical contribution to inflammatory responses. TG2 has been reported to increase the expression of proinflammatory cytokines through activation of the NF- $\kappa \mathrm{B}$ signaling pathway, including IL-6, TNF $\alpha$, IFN $\gamma$, IL-17A and IL-17F in mouse models of lipopolysaccharide or TNF $\alpha /$ actinomycin D-induced sepsis and bleomycin-induced lung fibrosis. ${ }^{10,11,29}$ Although expression levels of these cytokines in colon tissue are correlated with the severity of the DSS-triggered colitis, ${ }^{22}$ our results show that TG2 upregulates IL-6, inhibits IL-17A or has no effect on IL-17F and IFN $\gamma$ expression, indicating that TG2 differentially regulates cytokine expression in a tissue- or context-dependent manner.

Functionally, IL-6 is a proinflammatory cytokine, but it is also required for proliferation and survival of intestinal epithelial cells, ${ }^{37}$ contributing to the regeneration of injured epithelial cells in DSS-induced mouse colitis as well as human IBD. ${ }^{30,38,39}$ Thus, the high mortality rate of $\mathrm{TG}^{-/-}$mice may be attributed to lower expression levels of IL-6 compared with wild-type mice in response to DSS treatment. In addition, inconsistent with a previous report, ${ }^{9}$ TG2 ablation enhanced the expression of IL-17A and ROR $\gamma$ t despite low IL-6 levels, suggesting that IL-17A may play a different role in DSS-treated mice. Indeed, IL-17A has been reported to protect the gastrointestinal tract from injury by inducing genes that regulate mucosal barrier integrity, including claudins, proteins forming tight junctions. ${ }^{40}$ In support of these results, IL-17A ablation led to aggravation of DSS-induced colitis and $\mathrm{T}$ cell transfer-induced colitis in mice. ${ }^{41-43}$ Treatment with secukinumab, an IL-17A-specific blocking antibody, exacerbates Crohn's disease. ${ }^{44}$ Moreover, it should be noted that TG2 ablation also enhanced the expression of IL-10, an antiinflammatory cytokine for colitis in mice and humans. ${ }^{45-48}$ However, our data showed no difference in intestinal permeability and severity of inflammation between wild-type and $\mathrm{TG}^{-1-}$ mice. Hence, further investigation is required to ascertain the role of TG2-mediated inhibition of IL-17A and IL-10 in maintaining the intestinal epithelial barrier.

The enzymatic activity of TG2 is known to play a crucial role in eliciting an aberrant inflammatory response to dietary gluten peptide by deamidation and producing TG2 autoantibody in celiac disease. ${ }^{6}$ In contrast, our data demonstrate that intestinal 
TG2 is activated by DSS treatment, but is not involved in the tissue damage-induced inflammation. Thus, it will be worthwhile to investigate whether DSS treatment increases the level of TG2 autoantibody in the serum or the disease severity in the mouse model of celiac disease. This information might be important for understanding the exact role of TG2 in the pathogenesis of gluten-induced inflammation and developing therapeutics for celiac disease.

\section{CONFLICT OF INTEREST}

The authors declare no conflict of interest.

\section{ACKNOWLEDGEMENTS}

We thank the staff at the animal facility of Seoul National University College of Medicine for their help with animal breeding and care. This study was supported by grant of the National Research Foundation of Korea (NRF) funded by the Korea government (MSIP) (NRF-2014R1A2A2A01006731), Basic Science Research Program through NRF funded by the Ministry of Education (NRF-2013R1A1A2065273), the Korea Health Technology R\&D Project through the Korea Health Industry Development Institute (KHIDI) funded by the Ministry of Health \& Welfare, Republic of Korea (HI14C3339) and the Brain Korea 21 PLUS Program of the Korean Ministry of Education, Science and Technology. The funders had no role in study design, data collection and analysis, decision to publish or preparation of the manuscript.

1 lismaa SE, Mearns BM, Lorand L, Graham RM. Transglutaminases and disease: lessons from genetically engineered mouse models and inherited disorders. Physiol Rev 2009; 89: 991-1023.

2 Shin DM, Jeon JH, Kim CW, Cho SY, Kwon JC, Lee HJ et al. Cell typespecific activation of intracellular transglutaminase 2 by oxidative stress or ultraviolet irradiation: implications of transglutaminase 2 in age-related cataractogenesis. J Biol Chem 2004; 279: 15032-15039.

3 Siegel M, Strnad $P$, Watts RE, Choi K, Jabri B, Omary MB et al. Extracellular transglutaminase 2 is catalytically inactive, but is transiently activated upon tissue injury. PLOS ONE 2008; 3: e1861.

4 Jang GY, Jeon JH, Cho SY, Shin DM, Kim CW, Jeong EM et al. Transglutaminase 2 suppresses apoptosis by modulating caspase 3 and NF-kappaB activity in hypoxic tumor cells. Oncogene 2010; 29: 356-367.

5 Szondy Z, Korponay-Szabo I, Kiraly R, Fesus L. Transglutaminase 2 dysfunctions in the development of autoimmune disorders: celiac disease and TG2 ${ }^{-/-}$mouse. Adv Enzymol Relat Areas Mol Biol 2011 ; 78: 295-345.

6 Klock C, Diraimondo TR, Khosla C. Role of transglutaminase 2 in celiac disease pathogenesis. Semin Immunopathol 2012; 34: 513-522.

7 Sollid LM, Jabri B. Celiac disease and transglutaminase 2: a model for posttranslational modification of antigens and HLA association in the pathogenesis of autoimmune disorders. Curr Opin Immunol 2011; 23: 732-738.

8 Luciani A, Villella VR, Vasaturo A, Giardino I, Pettoello-Mantovani M, Guido $\mathrm{S}$ et al. Lysosomal accumulation of gliadin p31-43 peptide induces oxidative stress and tissue transglutaminase-mediated PPARgamma downregulation in intestinal epithelial cells and coeliac mucosa. Gut 2010; 59: 311-319.

9 Maiuri L, Luciani A, Giardino I, Raia V, Villella VR, D'Apolito M et al. Tissue transglutaminase activation modulates inflammation in cystic fibrosis via PPARgamma down-regulation. J Immunol 2008; 180: 7697-7705.

10 Falasca L, Farrace MG, Rinaldi A, Tuosto L, Melino G, Piacentini M. Transglutaminase type II is involved in the pathogenesis of endotoxic shock. J Immunol 2008; 180: 2616-2624.

11 Oh K, Park HB, Byoun OJ, Shin DM, Jeong EM, Kim YW et al. Epithelial transglutaminase 2 is needed for $T$ cell interleukin-17 production and subsequent pulmonary inflammation and fibrosis in bleomycin-treated mice. J Exp Med 2011; 208: 1707-1719.
12 Danese S, Fiocchi C. Ulcerative colitis. N Engl J Med 2011; 365: 1713-1725.

13 Baumgart DC, Sandborn WJ. Crohn's disease. Lancet 2012; 380: $1590-1605$

14 Van Limbergen J, Radford-Smith G, Satsangi J. Advances in IBD genetics. Nat Rev Gastroenterol Hepatol 2014; 11: 372-385.

15 Duerr RH, Taylor KD, Brant SR, Rioux JD, Silverberg MS, Daly MJ et al. A genome-wide association study identifies IL23R as an inflammatory bowel disease gene. Science 2006; 314: 1461-1463.

16 Barrett JC, Hansoul S, Nicolae DL, Cho JH, Duerr RH, Rioux JD et al. Genome-wide association defines more than 30 distinct susceptibility loci for Crohn's disease. Nat Genet 2008; 40: 955-962.

17 Jostins L, Ripke S, Weersma RK, Duerr RH, McGovern DP, Hui KY et al. Host-microbe interactions have shaped the genetic architecture of inflammatory bowel disease. Nature 2012; 491: 119-124.

18 Farrace MG, Picarelli A, Di Tola M, Sabbatella L, Marchione OP, Ippolito G et al. Presence of anti-'tissue' transglutaminase antibodies in inflammatory intestinal diseases: an apoptosis-associated event? Cell Death Differ 2001; 8: 767-770.

19 Okayasu I, Hatakeyama S, Yamada M, Ohkusa T, Inagaki Y, Nakaya R. A novel method in the induction of reliable experimental acute and chronic ulcerative colitis in mice. Gastroenterology 1990; 98: 694-702.

$20 \mathrm{Ni}$ J, Chen SF, Hollander D. Effects of dextran sulphate sodium on intestinal epithelial cells and intestinal lymphocytes. Gut 1996; 39 . 234-241.

21 Berndt BE, Zhang M, Chen GH, Huffnagle GB, Kao JY. The role of dendritic cells in the development of acute dextran sulfate sodium colitis. J Immunol 2007: 179: 6255-6262.

22 De Laurenzi V, Melino G. Gene disruption of tissue transglutaminase. Mol Cell Biol 2001; 21: 148-155.

23 Yan Y, Kolachala V, Dalmasso G, Nguyen H, Laroui H, Sitaraman SV et al. Temporal and spatial analysis of clinical and molecular parameters in dextran sodium sulfate induced colitis. PLOS ONE 2009; 4: e6073.

24 Livak KJ, Schmittgen TD. Analysis of relative gene expression data using real-time quantitative PCR and the 2(-Delta Delta C(T)) method. Methods 2001; 25: 402-408.

25 Eckert RL, Sturniolo MT, Broome AM, Ruse M, Rorke EA. Transglutaminase function in epidermis. J Invest Dermatol 2005; 124: 481-492.

26 Noti M, Corazza N, Mueller C, Berger B, Brunner T. TNF suppresses acute intestinal inflammation by inducing local glucocorticoid synthesis. J Exp Med 2010; 207: 1057-1066.

27 Ranganathan P, Jayakumar C, Manicassamy S, Ramesh G. CXCR2 knockout mice are protected against DSS-colitis-induced acute kidney injury and inflammation. Am J Physiol Renal Physiol 2013; 305: F1422-F1427.

28 Dafik L, Albertelli M, Stamnaes J, Sollid LM, Khosla C. Activation and inhibition of transglutaminase 2 in mice. PLOS ONE 2012; 7: e30642.

29 Yoo H, Ahn ER, Kim SJ, Lee SH, Oh SH, Kim SY. Divergent results induced by different types of septic shock in transglutaminase 2 knockout mice. Amino Acids 2013; 44: 189-197.

30 Naito Y, Takagi T, Uchiyama K, Kuroda M, Kokura S, Ichikawa H et al. Reduced intestinal inflammation induced by dextran sodium sulfate in interleukin-6-deficient mice. Int J Mol Med 2004; 14: 191-196.

31 Perse M, Cerar A. Dextran sodium sulphate colitis mouse model: traps and tricks. J Biomed Biotechnol 2012; 2012: 718617.

32 Kitajima S, Takuma S, Morimoto M. Tissue distribution of dextran sulfate sodium (DSS) in the acute phase of murine DSS-induced colitis. $J$ Vet Med Sci 1999; 61: 67-70.

33 Szondy Z, Sarang Z, Molnar P, Nemeth T, Piacentini M, Mastroberardino PG et al. Transglutaminase $2^{-/-}$mice reveal a phagocytosis-associated crosstalk between macrophages and apoptotic cells. Proc Natl Acad Sci USA 2003; 100: 7812-7817.

34 Balajthy Z, Csomos K, Vamosi G, Szanto A, Lanotte M, Fesus L. Tissue-transglutaminase contributes to neutrophil granulocyte differentiation and functions. Blood 2006; 108: 2045-2054.

35 Yin S, Gao B. Toll-like receptor 3 in liver diseases. Gastroenterol Res Pract 2010; 2010. pii: 750904.

36 Field R, Campion S, Warren C, Murray C, Cunningham C. Systemic challenge with the TLR3 agonist poly I:C induces amplified IFNalpha/beta and IL-1beta responses in the diseased brain and exacerbates chronic neurodegeneration. Brain Behav Immun 2010; 24: 996-1007.

37 Grivennikov S, Karin E, Terzic J, Mucida D, Yu GY, Vallabhapurapu S et al. IL-6 and Stat3 are required for survival of intestinal epithelial cells and development of colitis-associated cancer. Cancer Cell 2009; 15: 103-113. 
38 Sommer J, Engelowski E, Baran P, Garbers C, Floss DM, Scheller J. Interleukin-6, but not the interleukin-6 receptor plays a role in recovery from dextran sodium sulfate-induced colitis. Int J Mol Med 2014; 34: 651-660.

39 Mudter J, Neurath MF. IL-6 signaling in inflammatory bowel disease: pathophysiological role and clinical relevance. Inflamm Bowel Dis 2007; 13: $1016-1023$.

40 Kinugasa T, Sakaguchi T, Gu X, Reinecker HC. Claudins regulate the intestinal barrier in response to immune mediators. Gastroenterology 2000; 118: 1001-1011.

41 Ogawa A, Andoh A, Araki Y, Bamba T, Fujiyama Y. Neutralization of interleukin-17 aggravates dextran sulfate sodium-induced colitis in mice. Clin Immunol 2004; 110: 55-62.

42 Yang XO, Chang SH, Park H, Nurieva R, Shah B, Acero L et al. Regulation of inflammatory responses by IL-17F. J Exp Med 2008; 205: 1063-1075.

43 O'Connor W Jr, Kamanaka M, Booth CJ, Town T, Nakae S, Iwakura Y et al. A protective function for interleukin $17 \mathrm{~A}$ in $\mathrm{T}$ cell-mediated intestinal inflammation. Nat Immunol 2009; 10: 603-609.

44 Hueber W, Sands BE, Lewitzky S, Vandemeulebroecke M, Reinisch W, Higgins PD et al. Secukinumab, a human anti-IL-17A monoclonal antibody, for moderate to severe Crohn's disease: unexpected results of a randomised, double-blind placebo-controlled trial. Gut 2012; 61: 1693-1700.
45 Kuhn R, Lohler J, Rennick D, Rajewsky K, Muller W. Interleukin-10deficient mice develop chronic enterocolitis. Cell 1993; 75: 263-274.

46 Li B, Alli R, Vogel P, Geiger TL. IL-10 modulates DSS-induced colitis through a macrophage-ROS-NO axis. Mucosal Immunol 2014; 7: 869-878.

47 Correa I, Veny M, Esteller M, Pique JM, Yague J, Panes J et al. Defective IL-10 production in severe phenotypes of Crohn's disease. J Leukoc Biol 2009; 85: 896-903.

48 Franke A, Balschun T, Karlsen TH, Sventoraityte J, Nikolaus S, Mayr G et al. Sequence variants in IL10, ARPC2 and multiple other loci contribute to ulcerative colitis susceptibility. Nat Genet 2008; 40: 1319-1323.

(c) (i) (5) (2) This work is licensed under a Creative Commons Attribution-NonCommercial-ShareAlike $\quad 4.0$

International License. The images or other third party material in this article are included in the article's Creative Commons license, unless indicated otherwise in the credit line; if the material is not included under the Creative Commons license, users will need to obtain permission from the license holder to reproduce the material. To view a copy of this license, visit http://creativecommons.org/licenses/by-nc-sa/4.0/

Supplementary Information accompanies the paper on Experimental \& Molecular Medicine website (http://www.nature.com/emm) 